\title{
Prooftopia: A database for mathematical tools
}

\author{
Karina Figueroa M., Cuauhtémoc Rivera L., Luis \\ Valero E.
}

Published: 21 September 2016

\begin{abstract}
In this paper, we outline the current implementation of a database of mathematical theorems and their corresponding proofs which can be done via the web. We also provide a brief description of the current work for its complete redesign.
\end{abstract}

Keywords: Mathematical education; visualization; usability; online education

\section{Introduction}

Prooftopia is a database of mathematical theorems and proofs which can be accessed through a web. A mathematical proof is a deductive argument for a mathematical statement. In the argument, other previously established statements, such as theorems, can be used in the page [1].

It is interesting to point out that even without further work on its front end Prooftopia is still being used within the mathematical community in our school. It, however, demands an overhaul particularly in the way it is accessed. This is particularly evident with the broad availability of mobile devices and limited screen size real state that force the users to scroll extensively (both horizontal and vertically).

Originally, Prooftopia consisted of a single web page with two defined frames: a tutorial and the editor itself, writ- ten in PHP and basic HTML (Figures 1 and 2). They both make heavy use of frames, and focus primarily on the task of theorem proving without considering usability as a core component of the tools; the website does not include any CSS at all. Back when Prooftopia was created websites were used primarily on desktops computers whereas nowadays they are mainly accessed on mobile devices, which have intrinsically smaller displays.

There are several very positive points in the current implementation of Prooftopia: its coding is simple enough and well organized as in order to be adapted to more complex and user friendly iterations of the system.

Figueroa, K., Rivera, C., Valero, L.

Universidad Michoacana de San Nicolás de Hidalgo

Edificio Alfa, Ciudad Universitaria

Morelia, México

Email: karina@ fismat.umich.mx, crivera@ fismat.umich.mx,

valero@fismat.umich.mx

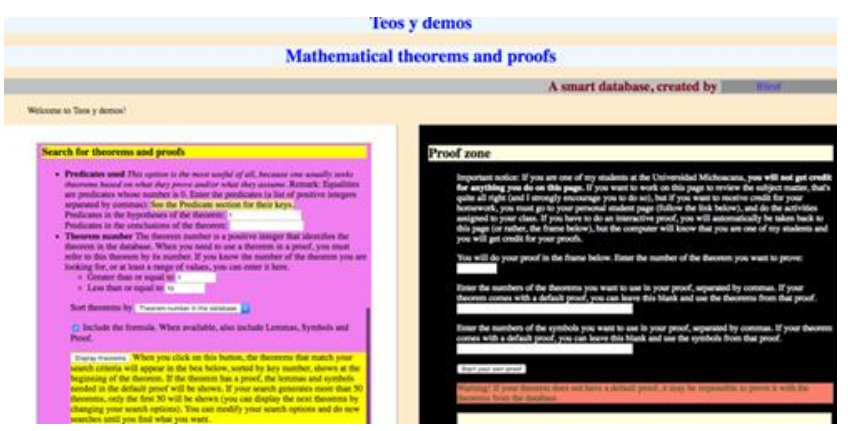

Figure 1. Current implementation of Prooftopia

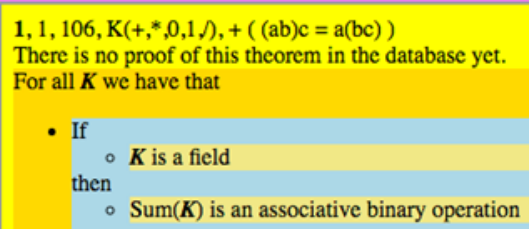

Figure 2. Theorem proving within Prooftopia

\section{Implementation details}

The original implementation of Prooftopia relies in a relational database, using MySQL for that purpose. All the user queries are handled with PHP scripts and the results are displayed within a corresponding frame in the Prooftopia web page (Figure 3 ).

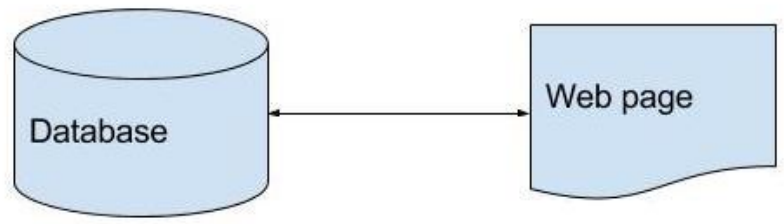

Figure 3. Current structure of Prooftopia 


\section{Justification}

There is an obvious need within the mathematical community for a set of tools as the one offered at Prooftopia, but to fully deliver its potential it has to be able to be success- fully used on different platforms. Particularly, it must be able to adapt to an ever changing hardware, with a particular focus on mobility. And whilst doing so, it must deliver a satisfactory user experience regardless of the platform used to access such tools.

\section{Current work}

The most urgent task is to make the entire set of tool HTML5 compliant, and fully separate the structure and style of it. It is also being considered to implement it with Bootstrap libraries which will allow us to make Prooftopia able to be render successfully in both mobile devices and traditional computers. Bootstrap [2] is one of the most widely supported frameworks used for developing responsive, mobile first projects on the web, which is perfect for our objective.

We envision Prooftopia as a real tool set for theorem proving, and as such, different sections in the current implementation will have to be put in standalone web pages. Modularization of the whole system is top priority in order to better maintain it and easily upgrade it when necessary. A very important aspect of our design include a recommender engine which would suggest additional resources (as in other web pages, courses, tutorials, and articles) to the users based on their performance with the system (figure 4) [3].

As it is, Prooftopia will be developed under strict usability design guidelines in order to ensure its optimum objective in helping the mathematical community with an easy to use mathematical proving system.

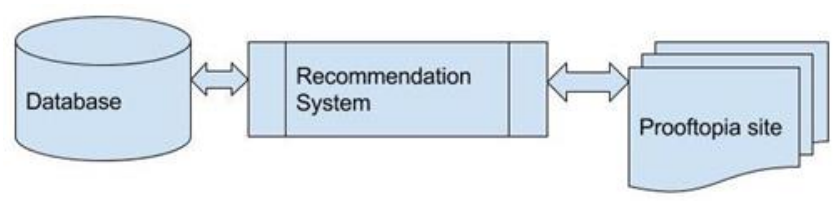

Figure 4. Basic architecture design for the new implementation of Prooftopia

\section{References}

[1] Clapham, C. and Nicholson, J.. 2009. "The concise oxford dictionary of mathematics". Oxford University Press.

[2] Moreto, Y. 2016. "Bootstrap By Example". Packt Publishing, New York, NY, USA, 1st edition,.

[3] Ricci, F., Rokach, L., Shapira, B. and Kantor, P. B. 2010. Recommender Systems Handbook. Springer-Verlag New York, Inc., New York, NY, USA, 1st edition. 